\title{
Trabajo Social y sus roles en el debate público sobre políticas sociales a través de los medios de comunicación en Puerto Rico
}

\author{
Social Work and its roles in public debate on social policies \\ throught the media in Puerto Rico
}

\author{
Elba BETANCOURT DÍAZ \\ Universidad de Puerto Rico \\ elba_betancourt@yahoo.com
}

Recibido: $20 / 06 / 2014$

Revisado: $31 / 07 / 2014$

Aceptado: 05/10/204

Disponible on line: 10/12/2014

\begin{abstract}
Resumen
El proceso de desarrollo, aprobación e implementación de políticas sociales está influenciado por la información que se difunde a través de los medios de comunicación. Por su preponderancia, estos medios se han convertido en un escenario más de acción política para los/las trabajadores/as sociales, quienes están convocados/as por los valores y códigos de ética profesionales a abogar por mejores políticas sociales para el bienestar de la sociedad. Sin embargo, su participación en el debate público sobre estas políticas sociales a través de los medios no ha sido particularmente estudiada. Esta investigación tiene como objetivo conocer los roles del Trabajo Social en el debate sobre las políticas sociales en los medios de comunicación en Puerto Rico. Utilicé un diseño exploratorio con método mixto secuencial, y en su fase cualitativa realicé entrevistas en profundidad semiestructuradas a 12 trabajadores/as sociales. En este artículo, esbozo solamente los hallazgos de este objetivo correspondiente a la etapa cualitativa. Del análisis de discurso surgió una dimensión vinculada a diversas funciones que pueden tener en el debate: mediadores/as, defensores/as o analistas/educadores/as. Finalmente, discuto las implicaciones que tienen esta inserción y estos roles en su desarrollo profesional.

Palabras clave: Trabajo Social, roles, medios de comunicación, políticas sociales, debate público.
\end{abstract}

\begin{abstract}
Social policy processes are influenced by news media content. For its importance these media outlets have become a scenario for political action for social workers, who are called to advocate for better social policies to promote social welfare, particularly for those populations their work with. However, their participation in the public debate through the media has been little studied. This research aimed to know the Social Work roles in public debate on social policies through the media in Puerto Rico. To conduct this study, I used a sequential exploratory mixed method design. In this article, I only exposed the findings regarding this objective corresponding to the qualitative phase, in which I conducted semistructured in-depth interviews to 12 Social Workers. From the analysis of discourse, emerged one dimension associated with possible functions of Social Work professionals in the debate, those were: mediators, advocates analysts/educators. Finally, I discuss the implications of their insertion and these roles in their professional development.
\end{abstract}

Keywords: social work, roles, media, social policy, public debate.

Referencia normalizada: Betancourt Díaz, E. (2014): «Trabajo Social y sus roles en el debate público sobre políticas sociales a través de los medios de comunicación en Puerto Rico». Cuadernos de Trabajo Social, 27(2): 417-427.

Sumario: Introducción. 1. Método. 2. Resultados. 3. Discusión. 4. Referencias bibliográficas.

\section{Introducción ${ }^{1}$}

Los mensajes divulgados a través de los medios de comunicación aportan información/desinfor- mación, y reproducen y perpetúan discursos sociales que influencian en los procesos de las políticas sociales. Por esto, los medios son para

${ }^{1}$ Los hallazgos aquí expuestos son parte de la investigación realizada como requisito para completar el grado doctoral en Trabajo Social, con especialidad en Política Social de la Universidad de Puerto Rico. 
los/as trabajadores/as sociales un escenario para influenciar la opinión pública, educando a la sociedad y, específicamente, a los/as hacedores/as de políticas públicas, a favor de políticas sociales más efectivas. Con una intervención adecuada estos/as, pueden dar visibilidad a problemáticas que permanecen soslayadas y contribuir a su transformación. Además, reafirman la noción de una profesión comprometida con su deber ético-político a nivel micro y macrosocial.

Los valores de justicia y equidad social en los que se fundamenta la profesión y su naturaleza política, convocan a los/as trabajadores/as sociales a ejercer su función de: comunicadores/as, intercesores/as, persuasores/as y colaboradores/as en los procesos de formulación y aprobación de políticas sociales (Guardiola, 2000). Estos roles, que se suman a otros que caracterizan a la profesión, surgen de su trabajo diario que les brinda una perspectiva particular de la realidad social y de las ventajas y desventajas de las políticas sociales. Esta es una fortaleza que les sirve para incursionar con éxito a nivel macro (Weiss-Gal y Peled, 2007), ahí donde se pueden influenciar las estructurales sociales generales que dan origen a muchos de los problemas sociales. De hecho, para que sus intervenciones logren transformaciones que disminuyan la inequidad y la marginación es necesario amalgamar las acciones a nivel macro con aquellas realizadas a nivel micro (Alayón, 2006).

Concebir y utilizar los medios como otra plataforma de acción requiere que los/as trabajadores/as sociales sepan cómo intervenir, en calidad de qué y para qué. La comunicación debe verse como una herramienta para aprovechar los reducidos espacios de libertad, un poder fundamental en las luchas sociales, y una manera de generar espacios contra la dominación y el sentido común imperante dentro de una hegemonía (Von Sprecher y Boito, 2010). Además, añaden estos autores citando a Gerbaldo (1999) que, cuando los/as comunicadores/as y/o trabadores/as sociales trabajan con clases subalternas, deben generar estrategias y planes para acceder a los medios y a la opinión pública para lograr que sus reclamaciones sean consideradas legítimas.

Este llamado se hace reconociendo que el acceso a los medios es asimétrico y que solo a algunos/as se les considera líderes/lideresas de opinión (Vallespín, 2000), lo que representa múltiples dificultades. Asimismo, se reconoce que los medios tradicionales suelen ser empresas privadas con intereses corporativos particulares o entidades públicas que responden a los intereses de las administraciones gubernamentales. Y en muchos casos estas dinámicas, en las que se interrelacionan poderes e intereses, se dan en un contexto de políticas económicas neoliberales que afectan al acceso a los medios, el mensaje que se difunde, la opinión pública y el desarrollo de políticas sociales. No obstante, los medios siguen siendo esa esfera pública donde se debate sobre política, ventilan ideas, construyen sentidos sobre los asuntos públicos y se difunden opiniones (Vallespín, 2000; Andrade del Cid, 2009). Esta función manifiesta la necesidad de identificar y comprender los roles que los/as trabajadores/as sociales pueden tener en esta dinámica de influencias sobre asuntos de política pública que a través de estos se suscita.

Sin embargo, la participación de los/as trabajadores/as sociales en los procesos de las políticas públicas en Puerto Rico no es muy frecuente y existe un desfase entre el deber de participar y concretar la acción (Guardiola Torres y Sierra Taylor, 2002; Canino-Arroyo et al., 2008). Por otro lado, a nivel internacional, la relación de estos profesionales con los medios la describen los estudios revisados como tensa por la representación negativa que se hace de los/as trabajadores/as sociales consideran, y por su falta de destrezas para manejar los medios según los/as periodistas (Freeman y Valentine, 2004; Zurazaga, Surette, Méndez y Otto, 2006; Gaughan y Garrett, 2011). Otra investigación revisada, apunta al fracaso de la profesión para influenciar la prensa y al público en el nivel de las ideas (Aldridge, 1990). Por otro lado, se han documentado los esfuerzos de las universidades para desarrollar en los/as alumnos/as destrezas para manejar los medios (Stone, Ekman, English y Fujimori, 2008; Weiss-Gal y Peled, 2009). Igualmente, encontré estudios que señalan la necesidad de desarrollar destrezas sobre el debate, argumentación, persuasión e intercesión mediática (Brawley, 1997; Curry-Stevens, 2001; Gregory y Holloway, 2005; Lens, 2005).

Al indagar en los roles del Trabajo Social existen estudios realizados en ámbitos como: el educativo (Concha Toro, 2012); el de la justicia (Conicella, Aveldaño y Aballay, 2011); el hospitalario (Bronstein, Kovacs y Vega, 2007; Craig y Muskat, 2013); y el de la gerontología (Koren y 
Doron, 2005; Naito-Chan, 2005); con familias en un contexto multidisciplinario (Dewees, 2004); con niños/as en centros de desarrollo temprano (Azzi-Lessing, 2010), con militares y sus familias (Manske, 2006). También se han investigado los roles en áreas como: programas para lidiar con la pobreza extrema (Saracostti, 2008); la administración de servicios sociales (Wuenschel, 2006); el ofrecimiento de servicios en áreas rurales (Saltman, Gumpert, Allen-Kelly y Zubrzycki, 2004); y las consideraciones psicosociales para la innovación de los roles (Conde Vieitez, 2003).

No obstante, no encontré datos específicos sobre los posibles roles o funciones que pueden realizar en el área de los medios. Para contribuir a la investigación sobre la relación entre el Trabajo Social y los medios, este estudio tiene como objetivo conocer los roles del Trabajo Social en el debate público sobre políticas sociales a través de los medios en Puerto Rico.

Esta investigación se fundamentó en el paradigma socioconstruccionista, que plantea que la realidad es una construcción colectiva que los/as individuos aprehenden a través de discursos que configuran una forma específica de interacción social (Berger y Luckmann, 1966). Bajo la mirada del socioconstruccionismo, se reconoce que la realidad es múltiple; se transforma mediante la construcción y reconstrucción de esos discursos; y se toman en cuenta múltiples voces que interactúan en la concepción de esa realidad.

Una de las maneras de legitimar los discursos, construir los imaginarios y naturalizar la realidad socioconstruida en las sociedades modernas, desarrolladas e industrializadas, es a través de los medios. McQuial (2000), esboza cinco metáforas que definen la mediación de los medios entre nosotros y la construcción social de la realidad: son una ventana para ver lo que ocurre; un espejo de los acontecimientos; un filtro que selecciona darle atención a solo partes de la realidad; una señal que explica lo que de otro modo sería fragmentado; una plataforma para la presentación de información e ideas; y una barrera que brinda sólo una visión falseada del mundo. De este modo, los medios funcionan como difusores discursivos, constructores y reproductores de imaginarios colectivos y naturalizan la realidad socialmente construida.

Aunque la literatura señala que es difícil determinar con precisión cuáles y cómo son los efectos de los medios en la sociedad, unos de los más documentados son los producidos por el establecimiento de una agenda de noticias: (agenda setting). Mediante este proceso el medio establece (de forma deliberada o no) la relevancia de un grupo de temas o eventos; el grado de prioridad o exposición que se les otorga; la repetición con la que se publican noticias sobre estos temas o eventos, y el contexto (framed) en el que se presentan (McCombs, 1997; Rubio Ferreres, 2009).

Los medios y la política forman parte de una dinámica de agendas que influencian a la opinión pública y el desarrollo de Políticas Sociales. Según Rogers y Dearing (1994), a la agenda de los medios se suman la agenda pública, que se relaciona con el contenido y orden de los temas en la opinión pública, y la agenda política, que se enfoca en la influencia de la agenda de los medios y la del público en los procesos de política pública. Los autores establecen que la agenda pública, una vez reflejada por la agenda de los medios, tiene influencia en la agenda de la política. Así como, la agenda de los medios parece tener una influencia directa sobre la agenda de la política pública y, en algunos asuntos, la agenda de la política pública parece tener una influencia directa sobre la agenda de los medios.

En este juego de influencias, los gremios también tienen una función. Si tomamos el Trabajo Social, observamos que diferentes códigos de deontología, incluyen en aspectos relacionados con acciones sociales y políticas que redundan en la justicia, equidad y bienestar social (CPTSPR, 2011; FITS y AITS, 2004; NASW, 2008). Específicamente, el Código de Ética del Colegio de Profesionales del Trabajo Social en Puerto Rico (2011) establece que el/la trabajador/a social: Se insertará en el análisis de los problemas y políticas sociales para ofrecer recomendaciones; fomentará la creación o los cambios en las políticas públicas que sean necesarios y estimulará que los/as participantes se inserten en los procesos de políticas públicas. La discusión pública de estos asuntos a través de los medios es una forma más de acción que tienen para como expone Rozas Pazaga (s.f.), «entrar al juego de los debates, de las posibilidades de incidir y alterar los criterios. Dicho de otro modo, es entrar al juego político en el que se pueda construir una hegemonía discursiva».

Si las política sociales se conciben como una definición estratégica de todo desarrollo, y un 
estructurador de la ciudadanía y de los derechos sociales siguiendo a esta autora, la intervención de los/as trabajadores/as sociales en cada etapa del proceso es inminentemente necesaria. Como menciona Guardiola (2002), el apoderamiento del proceso de formulación de política pública es una vuelta a las raíces políticas de la profesión. Bajo esta conceptualización ideológica de la profesión, se denuncia el orden existente mediante la concienciación y movilización de la gente a través de la acción social, autogestión, gestión pública y la participación del pueblo en el ámbito político, económico y social (Guardiola, 1995). Esta participación es fundamental, sobre todo en el contexto de las políticas de corte neoliberal, que promueven un grado de mercantilización de los bienes sociales y someten su prestación a una lógica de lucro, coartando el ejercicio pleno de la ciudadanía.

Por otro lado, si agregamos el uso de los medios como una estrategia más de acción política, Chinchilla (2002) expone que el nivel de incapacidad que demuestra la profesión para insertarse en la dinámica de los medios puede deberse a que no ha sido constante la participación sistemática de la profesión en los movimientos sociales (aunque sí ocurra a nivel individual). Al respecto, propone que el reto del Trabajo Social para utilizar con éxito y de forma sostenida los medios, depende de que realicen «un trabajo de sensibilización, información, promoción, creación de opinión pública y construcción de poder no solo desde la base profesional, sino particularmente desde las estructuras sociales». (p. 12)

Desde este punto de vista, los/as trabajadores/as sociales tienen que afianzar sus vínculos con las estructuras sociales, en la que se incluyen los medios, y entender sus posibles roles para que su participación sea una estratégica que les acerque más a la transformación social a la que aspiran. En palabras de Brawley (1997), como los medios influencian en la opinión pública y son una fuente de información para los/as hacedores/as de política pública y el público, quienes promueven la justicia social y económica necesitan ser participantes activos/as en el proceso de agenda setting, construcción de la opinión pública y formulación de políticas.

\section{Método}

Para realizar el estudio utilicé un diseño de métodos mixtos (Creswell, 2003). Para el propósi- to de este artículo me limitaré a detallar el método de la fase cualitativa, de la que surgieron los resultados que presento. En esta etapa, realicé entrevistas en profundidad semiestructuradas a 12 trabajadores/as sociales, seis que habían participado en el debate público sobre políticas sociales a través de los medios y seis que no habían participado.

\subsection{Participantes}

La muestra fue una por disponibilidad compuesta por 12 trabajadores/as sociales mayores de edad, seis que habían participado del debate público a través de los medios y seis que no. Los/as seis primeros fueron identificados/as durante el análisis de contenido que realicé en la fase cuantitativa de este estudio. Invité a participar, mediante carta, a los seis que más aparecieron entrevistados/as o como autores/as de artículos de opinión. Todos/as aceptaron ser entrevistados/as. Estos/as profesionales provenían de la academia y/o habían ocupado puestos de liderazgo en el Colegio de Profesionales del Trabajo Social de Puerto Rico (CPTSPR) o en una dependencia gubernamental.

Los/as seis que no habían participado en el debate público fueron reclutados/as a través de material promocional en las escuelas graduadas de Trabajo Social de universidades del área metropolitana, en el Colegio de Profesionales del Trabajo Social y en el Departamento de la Familia (Agencia Gubernamental encargada de los Servicios Sociales). Se completó con una descripción del grado académico, años de experiencia profesional y puesto que ocupaba de cada participante (ver Tabla 1).

\subsection{Procedimiento}

El protocolo de investigación fue autorizado por el Comité Institucional para la Protección de los Seres Humanos en la investigación de la Universidad de Puerto Rico. Tras la aprobación, inicié el reclutamiento. Después de evaluar que cumplían con los criterios de inclusión, y de haberles explicado el propósito del estudio, coordiné las entrevistas. Todos/as los/as entrevistados/as firmaron la hoja de consentimiento. A los/as participantes que no habían participado del debate, les ofrecí confidencialidad; mientras, que a las personas identificables por sus temas de trabajo o exposición en los medios, les expliqué que no les aseguraba esta confidencia- 


\begin{tabular}{|l|l|c|l|}
\hline $\begin{array}{c}\text { Trabajador/a } \\
\text { Social }\end{array}$ & \multicolumn{1}{c|}{ Grado } & Años de experiencia & \multicolumn{1}{|c|}{ Puesto que ocupa } \\
\hline TS1P & Doctorado Sociología & 37 & Retirado \\
\hline TS2P & Doctorado TS & 25 & Catedrática \\
\hline TS3P & Doc. Sicología (en progreso) & 15 & Catedrático \\
\hline TS4P & Doctorado TS & Más de 30 & Práctica Privada \\
\hline TS5P & Doctor Derecho & 37 & Catedrática \\
\hline TS6P & Maestría TS y Adm. Escolar & 28 & TS Escolar \\
\hline TS7NP2 & Maestría TS & 12 & TS Salud Mental \\
\hline TS8NP & Maestría Orientación & 22 & TS Depto. Familia \\
\hline TS9NP & Bachillerato TS & 15 & TS Depto. Familia \\
\hline TS10NP & Maestría TS (en progreso) & 5 & TS Depto. Familia \\
\hline TS11NP & Maestría TS & 3 & Experta de Caso \\
\hline TS12NP & Doc. Psicología (en progreso) & 5 & Interna en Psicología \\
\hline
\end{tabular}

Tabla 1. Descripción de los/as participantes.

1 TS1P: se refiere a Trabajadores/as Sociales que participaron del debate y el orden de la entrevista.

2 TS2NP: se refiere a Trabajadores/as Sociales que no participaron del debate y el orden de la entrevista.

lidad. Las entrevistas fueron grabadas y duraron de 20 minutos a una hora. Para asegurar la confidencialidad utilicé códigos numéricos en la hoja de consentimiento, grabaciones y transcripciones.

\subsection{Instrumento}

Para recopilar los datos usé dos guías de preguntas: una dirigida a las personas que habían participado del debate y otras a las que no habían participado. Las preguntas abiertas giraron en torno a: experiencias de participación en el debate sobre política sociales a través de los medios; posibles roles de la profesión en el debate, factores individuales que fomentan la participación; factores estructurales que obstaculizan la participación y estrategias para potenciar la participación.

\subsection{Análisis}

Transcribí las entrevistas a verbatium utilizando el programado HyperTranscribe para el posterior análisis de discurso. Inicié el proceso de análisis haciendo anotaciones sobre ideas, patrones y temas que surgieron en las entrevistas (Potter, 2009). Posteriormente, desarrollé dimensiones temáticas y categorías que me facili- taran trabajar con una gran cantidad de datos textuales (Potter y Wetherell, 1987; Potter, 2009). A continuación una descripción de la dimensión analítica amplia y las categorías que surgieron del análisis (ver Tabla 2).

\section{Resultados}

Del análisis surgió una dimensión analítica amplia que agrupa asuntos que emanaron de las entrevistas relacionadas con la función que los/as participantes le reconocieron al Trabajo Social en el debate público sobre PS a través de los medios. Estos hallazgos son interesantes porque los/as entrevistados/as verbalizaron que el/la profesional del Trabajo Social puede ejercer más de un rol en este debate. La forma en que conciben su participación arroja luz sobre cómo pueden insertarse al debate público sobre PS y cómo los medios noticiosos pueden incluirlos/as en sus trabajos. Del análisis surgieron tres (3) categorías: (1) mediadores/as; (2) defensores/as; y (3) analistas/educadores. A continuación detallo las categorías e incluyo algunas verbalizaciones.

Mediadores/as. Algunos/as entrevistados/as consideraron que pueden ser un vínculo entre los/as periodistas y las poblaciones, comunidades o individuos/as involucrados/as en el tema o 


\begin{tabular}{|c|c|}
\hline Dimensión analítica amplia & Categorías \\
\hline $\begin{array}{l}\text { Función del Trabajo Social en el de- } \\
\text { bate público } \\
\text { Esta categoría apunta a posibles roles } \\
\text { de los/as trabajadores/as sociales en el } \\
\text { debate público sobre Política Social. }\end{array}$ & $\begin{array}{l}\text { Mediadores/as. Expresiones sobre cómo pueden servir de } \\
\text { vínculo entre los/as periodistas y las poblaciones, comunida- } \\
\text { des o individuos involucrados/as en el tema o política social } \\
\text { en discusión. } \\
\text { Defensores/as. Expresiones sobre participar del debate a fa- } \\
\text { vor de las poblaciones, comunidades o individuos con los/as } \\
\text { que trabajan. } \\
\text { Analistas/educadores/as. Expresiones sobre contribuir con } \\
\text { sus conocimientos y experiencias a la comprensión de los te- } \\
\text { mas, casos particulares o fenómenos sociales, y el desarrollo, } \\
\text { implementación y evaluación de Política Social. }\end{array}$ \\
\hline
\end{tabular}

Tabla 2. Descripción de la dimensión y las categorías.

política social en discusión. Además, expresaron que pueden abonar al debate público sus experiencias trabajando con estas poblaciones, comunidades o individuos involucrados/as y sus realidades sociales.

Yo considero que los trabajadores sociales somos un grupo profesional que tenemos mucho que contribuir porque, eh, la experiencia misma. Estamos tan cerca de las clases más necesitadas. Estamos bien cerca de la realidad del país. Eh, y hemos acumulado una experiencia, ¿verdad?, y una visión de lo que es realmente la realidad de Puerto Rico que tenemos la responsabilidad de contribuir al expresarla para que terceros así la conozcan (TS5-P).

Bueno, [piensa] quizás, entiendo yo que el Trabajador Social pudiera ser este ente que le permite a la personas ver lo que realmente está sucediendo en la calle. Tú sabes no es lo mismo verlo de lejos o de oídas, escuchar de oídas, que tener un trabajador social que constantemente está atendiendo al público, ya sea dentro de la oficina o fuera de la oficina (TS10-NP).

Sería como el intermediario entre el individuo y la comunidad y el espacio público [...]. (TS11-NP)

Estas verbalizaciones aluden a que los medios, al reportar sobre temas sociales, no necesariamente presentan la realidad tal como los/as trabajadores/as sociales la aprecian a través de sus intervenciones cotidianas. Por esto, expresaron que ellos/as pueden colaborar con los medios como un enlace o intermediarios/as que les permita a los/as periodistas conocer y comprender mejor la realidad de los/as ciudadanos/as. Este ejercicio de mediador/a daría oportunidad a los/as individuos, grupos y comunidades a expresarse sobre los problemas sociales que les aquejan añadiendo posibles soluciones al debate.

Defensores/as. Los/as entrevistados/as identificaron que pueden participar expresándose a favor de las poblaciones, comunidades o individuos con los/as que trabajan para promover agendas particulares que redunden en políticas sociales más efectivas.

PART: [...] por un lado deberíamos ser abogados o intercesores, por otro lado deberíamos ser personas que ofrecen alternativas. Y a eso, ¿cómo le podemos llamar? Alguna persona que resuelva conflicto como le podemos... el concepto para roles... mediador o que el otro también es intercesor. A la larga, que no se quede solo en la denuncia nuestro rol también: tratar de traer alternativas (TS2-P).

Yo creo que los trabajadores y trabajadoras sociales tenemos un rol de, yo le llamaría crítico, eh, por el tipo de población que se supone que el trabajo social sirva. Históricamente, el Trabajo Social es una profesión vinculada a poblaciones marginadas, a las poblaciones de alguna forma oprimida [...]. Esto hace necesario la presencia de los trabajadores sociales en los medios precisamente como una especie de voces de esas poblaciones. Yo recuerdo siempre el apodo que le decían al Monseñor Romero en El Salvador: La voz $\sin$ los sin voz. Un poco, verdad, retomando ese tipo de metáfora algo así debería ser la función 
del Trabajo Social en los medios. Hacer presencia en defensa, en búsqueda de alternativas para la solución de los problemas (TS3-P).

Bueno, sí, porque nosotros tenemos que abogar en favor de nuestra clientela que muchas veces son personas que están en rezago cultural, que son personas que obviamente no tiene el acceso ni a los medios ni la capacidad de comunicación. Y podemos ser quienes podamos llevar la voz de esas comunidades que atendemos para que a través de nosotros se dejen sentir y se tomen en cuenta (TS9-NP).

Estas verbalizaciones manifiestan la responsabilidad de ser la voz de las poblaciones que atienden, realizando una labor de intercesoría y defensa que denuncie las estructuras que perpetúan la injusticia y la inequidad social. Como expresó una de las participantes: muchos/as de estas poblaciones o individuos no tienen las posibilidades o destrezas para conseguir ser escuchados/as, por lo que el/la trabajador/a social viene llamado/a a ser su intercesor/a público. La inclusión de estas voces enriquece la discusión y contribuye al desarrollo de políticas más enfocadas en la realidad social que ellos/as viven.

Analistas/educadores/as. De acuerdo con algunos/as entrevistados/as, pueden aportar sus conocimientos y experiencias, como implementadores/as de políticas sociales, a la comprensión de los fenómenos sociales y al análisis de las políticas sociales. Además, pueden ayudar a que los/as periodistas comprendan los temas sociales que cubren, mostrarles posibles ángulos para trabajar sus historias y ayudarles a analizar el impacto de las políticas sociales.

Podríamos ser analistas de lo que sea la realidad social, problemas sociales, fenómenos sociales y, obviamente, dentro del contexto de la política social (TS2-P).

Cada vez que él [hace referencia a un periodista] va a escribir él me llama y me pide, lo que él llama, una opinión informada de un experto para ver cómo él puede enfocar o analizar el artículo. Y yo siempre insisto en la dimensión crítica [...]. O sea, que yo estoy haciendo como un papel desde mi perspectiva de trabajo social, ¿verdad?, y el conocimiento que yo he acumulado, de orientar al desarrollo de noticias de un periódico con el cual yo no tengo ningún vínculo. [...] yo me voluntarié para esto, porque entiendo que como profesional de Trabajo Social esto era una de mis funciones [...]. A veces me doy cuenta, como educador en este momento, educador de un periodista o de algunos periodistas, que se les asignan tareas para las cuales no tienen educación formal, lo que me hacía difícil a mí la explicación para que ellos la entendieran (TS1-P).

PART: Mi participación ha sido fundamentalmente a través de la crítica de políticas específicas. Eh, no necesariamente políticas sociales en la práctica, [...], sino más bien el contenido, la visión que emerge el discurso ideológico que hay detrás de las políticas sociales que se han aprobado o implantado en Puerto Rico y también en los Estados Unidos (TS3-P).

Pues, mira, yo pienso que el trabajador social puede llevar esa dosis o esa parte de la información que es más de análisis del beneficio a la gente, o sea, fuera de la cuestión puramente [político] partidista que muchas veces se da en ese discurso. (TS5-P)

Estas verbalizaciones apuntan al valor que pueden tener las aportaciones los/as trabajadores/as sociales a la discusión de fenómenos y políticas sociales, porque pueden hacer un análisis particular desde su perspectiva profesional. Estos/as pueden con sus análisis señalar públicamente posibles consecuencias de la implementación de ciertas políticas sociales. Asimismo, con su análisis pueden educar a la población y a los/as periodistas, pues aportan otros puntos de vistas más críticos y temáticas de interés. Su intervención sirve para guiar la discusión sobre políticas sociales por otros ámbitos que a veces son ignorados porque otros discursos, como los políticos partidistas, son más predominantes.

\section{Discusión}

El deber y la necesidad de que los/as trabajadores/as sociales sean parte del debate público sobre políticas sociales a través de los medios han sido reconocidos por la literatura, los códigos de ética a nivel nacional e internacional, y los/as entrevistados/as de este estudio. Estos/as últimos/as incluso identificaron varios roles específicos que pueden tener basándose en sus conocimientos y experiencias profesionales. De acuerdo con ellos/as, pueden desempeñarse co- 
mo: intercesores/as, mediadores/as y analistas de políticas sociales.

Como mediadores/as pueden ser ese enlace con los/as profesionales de los medios para contribuir a presentar la realidad social en una dimensión más completa y compleja de lo que usualmente se presenta. El rol de defensores/as puede contribuir a que las poblaciones marginadas y excluidas tengan una voz fuerte y activa en los medios que interceda por ellos/as y les brinde visibilidad. Como analistas/educadores/as pueden aportar sus puntos de vistas sobre las políticas sociales enriqueciendo el debate y dirigiendo esta discusión fuera del terreno político partidista que suele acaparar estas temáticas. Estos roles resultan ser inherentes al ejercicio de la profesión, pues están acorde con sus fundamentos y principios. Asimismo, deben darse en el nivel marco de intervención junto a sociedades civiles que cada vez más busca las formas de influir los procesos de desarrollo e implementación de las políticas sociales.

No obstante, como menciona Matus-Sepúlveda (2002), la mediación para que sea transformadora no puede ser solo para hablar de otros/as, sino para mostrar las contradicciones de los discursos en sociedades donde se pretende mostrar un patrón uniforme de comportamiento y lo que se desvíe se considera transgresión. En palabras de esta trabajadora social: «La función mediadora de Trabajo Social debe contener la posibilidad de recuperar la unidad de lo razonable dejando escuchar sus múltiples voces». A nivel macrosocial, el análisis de las políticas sociales proveniente del Trabajo Social a debe aportar una mirada a veces ausente del debate público. Como establece la autora, el profesional del Trabajo Social puede evaluar y analizar las políticas, no solo tomando en cuenta su cobertura, sino también mostrando las experiencias de los/as sujetos beneficiarios/as, la calidad con la que se les ofrecen los servicios, y develando e interpretando las contradicciones entre los objetivos de la política, su ejecución y evaluación. Esta tarea requiere de la realización de investigaciones que aporten los datos y evidencias necesarios para intervenir en el nivel macrosocial generando un análisis distinto al que estamos acostumbrados/as, el cual que redunde en mejores políticas y prácticas sociales.

Otra forma de promover una discusión diferente es utilizando los principios de la interceso- ría mediática, la cual propone mover la discusión de una individualista a una universal. Como explican Wallack et al. (1993) desde una visión individualista los problemas son causados por asuntos personales o de estilos de vida y se cae en el error de culpar a las víctimas. Mientras, que en el enfoque universalista, los problemas son definidos y entendidos desde los factores estructurales o condiciones sociales que los provocan, lo que redirige la discusión al terreno de las macroestructuras y las políticas sociales.

Estos hallazgos también tienen implicaciones en la formación y socialización de estos/as profesionales. Específicamente, brindan una idea sobre cómo dirigir los esfuerzos de educación de los/as futuros/as trabajadores/as sociales sobre para qué y cómo insertarse en el debate público sobre políticas sociales a través de los medios. Estas iniciativas deben enfatizar el vínculo de este otro escenario de acción, las políticas sociales, los roles que pueden asumir y la necesidad de sus aportaciones. De igual forma, deben desarrollar destrezas que faciliten la inserción y práctica de estos/as en el debate público y en los medios. Por otro lado, estos resultados sirven para que los medios de comunicación tomen en cuenta cómo pueden incluir a estos/as profesionales en sus trabajos periodísticos para enriquecerlos y cumplir a cabalidad su responsabilidad social.

Finalmente, invitan a reflexionar sobre por qué la identificación de roles, así como el reconocimiento de la importancia de su participación en el nivel macrosocial, no necesariamente implica que se inserten en el debate a través de los medios. En esta discusión se deben tomar en cuenta obstáculos estructurales e individuales vinculados a su posible participación como: su socialización durante sus años de formación académica y su desarrollo profesional; las dinámicas de poder al interior de sus trabajos; y su interacción con los medios, que median en su decisión de participar (Betancourt Díaz, 2014). Estos obstáculos contribuyen al desfase entre la teoría y la práctica; entre el deber, el querer y el poder; y entre los posibles niveles de intervención: micro, meso y macro. Sobrepasarlos es fundamental para que los/as trabajadores/as sociales ejerzan sus posibles roles en el debate sobre política sociales a través de los medios de manera efectiva reforzando su compromiso ético-político a nivel macrosocial. 


\section{Referencias bibliográficas}

Alayón, N. (2006). Democracia, derechos sociales y trabajo social. En N. Burgos Ortiz (ed.), Política social y trabajo social. San Juan: Proyecto Atlantea.

Aldridge, M. (1990). Social Work and News Media: A Hopeless Case? The British Journal of Social Work, 20 (6), 611-625.

Andrade del Cid, P. (2009). La democracia en el espacio público mediático. Global Media Journal Edición Iberoamericana, 6 (12), 91-100.

Azzi-Lessing, L. (2010). Growing Together: Expanding Roles for Social Work Practice in Early Childhood Settings. Social Work, 55(3), 255-263.

Berger, P. y Luckman, T. (1966). The Social Construction of Reality. USA: Anchor Books.

Betancourt Díaz, E. (2014). Los medios de comunicación como escenario de debate sobre políticas sociales: Implicaciones para el Trabajo Social en Puerto Rico. (Tesis Doctoral sin publicar). Universidad de Puerto Rico, Río Piedras.

Brawley, E. A. (1997). Teaching social work students use advocacy skills through the mass media, Journal of Social Work Education, 33, 445-60.

Bronstein, L., Kovacs, P. y Vega, A. (2007). Goodness of Fit. Social Work in Health Care, 45 (2), 59-76.

Canino Arroyo, M.J., Lugo Hernández, E., Díaz Meléndez, L., Vélez Pérez, D., Carvallo Messa, V. y Serrano García, I. (2008). Encuesta de la participación de trabajadores y trabajadoras sociales en la política pública en Puerto Rico. En Política Social y Trabajo Social: Comunidades y políticas sociales entre la académica y la práctica cotidiana. 4, 67-87.

Chinchilla Montes, M. (2002). Poder, medios de comunicación social y Trabajo Social. Ponencia presentada en el III Congreso Anual de la Federación Nacional de Estudiantes y Egresados de Trabajo Social. Culiacán, México.

Concha Toro, M. (2012). Rol, perfil y espacio profesional del Trabajo Social en el ámbito educativo. Cuadernos de Trabajo Social, 5,11-126. Recuperado de: http://trabajosocial.blogutem.cl/files/2011/07/Cuadernos_Trabajo_Social_R5.pdf.

Conde Vieitez, J. (2003). El rol del trabajador social: consideraciones psicosociales para la innovación de rol. Cuadernos de Trabajo Social, 16, 73 - 91.

Conicella, V., Aveldaño, V. y Aballay, P. (2011). Nuevos escenarios de actuación profesional: el Trabajo Social y la Justicia. Debate Público. Reflexión de Trabajo Social, 1 (2), 63-71.

CPTSPR. (2011). Código de Ética de Trabajo Social. San Juan: Autor.

Craig, S. L. y Muskat, B. (2013). Bouncers, brokers, and glue: The self-described roles of social workers in urban hospitals. Health \& Social Work, 38 (1), 7-16. Recuperado de: http://search.proquest.com/docview/1328997081? accountid $=44825$

Creswell, J. (2003). Research Design Qualitative, Quantitative and Mixed Methods Approaches. Thousand Oaks, CA: Sage.

Curry-Stevens, A. (2012). Persuasion: Infusing Advocacy Practice with Insights from AntiOppression Practice, Journal of Social Work, 12(4), 345-363. Recuperado de: http://ejournals.ebsco.com/direct.asp?ArticleID=4A1DB65DFBB3384765B2.

Dewees, M. (2004). Postmodern Social Work in Interdisciplinary Contexts. Social Work in Health Care, 39 (3-4), 343-360. Recuperado de: http://ejournals.ebsco.com/direct.asp?ArticleID= 4DDBA224799AC4B806C3.

FITS y AETS. (2004). Código de Ética. Recuperado de: http://www3.unileon.es/ce/ets/ficheros/Codigo_FITS.pdf.

Freeman, M. y Valentine, D. (2004). Through the Eyes of Hollywood: Images of Social Workers in Film. Journal Social Work, 49 (2), 151-161.

Gaughan, L. y Garrett, P. (2012). The most twisted and unaccountable force in the state? Newspaper accounts of social work in the Republic of Ireland in troubled times. Journal of Social Work, $12,267-286$.

Gregory, M. y Holloway, M. (2005). The Debate as a Pedagogic Tool in Social Policy for Social Work Students. Social Work Education, 24 (6), 617-637. 
Guardiola, D. (1995). Trabajo Social en Puerto Rico: ¿Asistencialismo, desarrollismo o transformación? Ponencia presentada en la Escuela Graduada de Trabajo Social Beatriz Lassalle. San Juan, Puerto Rico.

Guardiola, D. y Sierra Taylor, J. (2002). Política social y trabajo social en Puerto Rico. Desafio y alternativas para el siglo XXI. San Juan: Publicaciones Puertorriqueñas.

Koren, C. y Doron, I. (2005). Being a Social Worker in Homes for the Aged. Journal of Gerontological Social Work, 44 (3-4), 95-114. Recuperado de: http://ejournals.ebsco.com/direct.asp?ArticleID=43979E510D7E0383BA08.

Lens, V. (2005). Advocacy and Argumentation in the Public Arena: A Guide for Social Workers. Social Work, 50 (3), 231-238.

Manske, J. E. (2006). Social Work in the Department of Veterans Affairs: Lessons Learned. Health \& Social Work, 31 (3), 233-238. Recuperado de: http://search.proquest.com/docview/2 10560171 ? accountid $=44825$.

Matus-Sepúlveda, T. (2002). Propuestas contemporáneas en trabajo social: hacia una intervención polifónica. Buenos Aires: Espacio.

McCombs, M. (1997). Building Consensus: The News Media's Agenda Setting Roles. Political Communication, 14, 433-443.

McQuial, D. (2000). Introducción a la teoría de la comunicación de masas. Barcelona: Paidós.

Mendes, P. (2001). Blaming the Messenger: The Media, Social Workers and Child Abuse. The Journal of the Australian Association of Social Workers, 54 (2), 27-36.

Naito-Chan, E. (2005). Identifying Competencies for Geriatric Social Work Practice. Journal of Gerontological Social Work, 43 (4), 59-78. Recuperado de: http://ejournals.ebsco.com/direct. asp?ArticleID=466E91C3D6469C6A39E6.

Potter, J. (2009). Discourse Analysis. En: M. Hardy y A. Bryman (eds.), Handbook of Data Analysis (pp. 607-624). Londres: Sage.

Potter, J. y Wetherell, M. (1987). Discourse and Social Psychology: Beyond Attitudes and Behavior. Londres: Sage.

Rozas Pagaza, M. (s.f.). La intervención profesional, politicas sociales y derechos sociales claves para la construcción de la ciudadanía. Recuperado de: http://www2.fices.unsl.edu.ar/ $\sim$ prosoc/documentos/La_intervencion.doc.

Rogers, E. y Daring, J. (1994). Agenda setting research: where has it been, where is it going? En: D. Graber (Ed.), Media Power in Politics (pp.77-95). Washington, DC: CQ Press.

Rubio Ferreres, J. (2009). Opinión pública y medios de comunicación. Teoría de 1 agenda setting. Gazeta de Antropología, 25 (1).

Saltman, J., Gumpert, J., Allen-Kelly, K. y Zubrzycki, J. (2004). Rural social work practice in the United States and Australia: A comparison. International Social Work, 47 (4), 515-531. Recuperado de: http://ejournals.ebsco.com/direct.asp?ArticleID=47A9BC38920386BC21CC.

Saracostti, M. (2008). The Chile Solidario system: The role of social work. International Social Work, 51 (4), 566-572. Recuperado de: http://ejournals.ebsco.com/direct.asp?ArticleID= 4EBDBC18ABBE6967F882.

Stone, S., Ekman, E., English, D. y Fujimori, S. (2008). Collaboration among Social Work and Journalism Students and Faculty. Journal of Social Work Education, 44 (1), 163-172.

Vallespín, F. (2000). La crisis de espacio público. Revista Española de Ciencias Políticas, 3,77-95.

Von Sprecher, R. y Boito, M.E. (2010). Comunicación y trabajo social. Córdova, Argentina: Editorial Brujas.

Wallack, L., Dorfman, L., Jernigan, D. y Themba, M. (1993). Media Advocacy and Public Health. California: Sage Publications.

Weiss Gal, I. y Peled, E. (2007). Publishing Voice: Training Social Workers in Policy Practice. British Journal of Social Work, 39 (2), 368-382.

Wuenschel, P. (2006). The Diminishing Role of Social Work Administrators in Social Service Agencies. Administration in Social Work, 30(4), 5-18. Recuperado de: http://ejournals.ebsco.com/direct.asp?ArticleID=4931B7A325155E8BD396. 
Zurazaga, C., Surette, R., Mendez, M. y Otto, C. (2006). Social Worker Perceptions of the Portrayal of the Profession in the Media. Journal of Social Work Education, 42 (3), 621-636. 